\title{
Expanding the Scope of a Dynamic Perspective on Positive Adolescent Sexual Development
}

\section{Commentary on Arbeit}

Lisa M. Diamond

University of Utah, Salt Lake City, Utah, USA

\author{
Key Words \\ Adolescents · Dynamic perspective $\cdot$ Sexual development
}

The World Health Organization (WHO) convened a meeting on sexual health in 2002 to clarify definitions of healthy sexual development and concluded with a powerful statement testifying to the central and multifaceted role of sexuality in human health and well-being [World Health Organization, 2004]. According to their model, sexuality incorporates eroticism, intimacy, pleasure, reproduction, and one's own gender identity, and it is manifested in sexual behaviors, wishes, desires, fantasies, attitudes, roles, and relationships. The WHO also views sexuality as inherently multidimensional, integrating biological, cultural, social, economic, psychological, ethical, and spiritual factors. Positive or healthy sexuality, then, requires that individuals have adequate freedom and knowledge to pursue safe and satisfying sexuality. In the compelling words of the WHO [2004]:

Sexual health is a state of physical, emotional, mental and social well-being in relation to sexuality; it is not merely the absence of disease, dysfunction or infirmity. Sexual health requires a positive and respectful approach to sexuality and sexual relationships, as well as the possibility of having pleasurable and safe sexual experiences, free of coercion, discrimination and violence. (p. 3)

A decade after the publication of this powerful statement, the study of adolescent sexuality remains more focused on negative than positive experiences. Although a number of scholars have advocated for positively-oriented conceptualizations of adolescent sexuality [Russell, 2005; Tolman \& McClelland, 2011], the majority of developmental research continues to view adolescent sexuality through a lens of danger and threat, overemphasizing the timing and frequency of risky sexual acts and their negative implications for future health and adjustment. We remain woefully uninformed

\begin{tabular}{ll}
\hline KARGER & ○ 2014 S. Karger AG, Basel \\
$\begin{array}{l}\text { E-Mail karger@karger.com } \\
\text { www.karger.com/hde }\end{array}$ &
\end{tabular}

Lisa M. Diamond

Department of Psychology, University of Utah

380 South 1530 East, Room 502

Salt Lake City, UT 84112 (USA)

E-Mail diamond@psych.utah.edu 
about youths' total psychological and physical experience of erotic and affectional desires, cognitions, and behaviors from childhood to adulthood and their potentially positive developmental implications for notions of self, identity, and relationships.

For these reasons, Miriam Arbeit's [this issue] skill-based model for promoting adolescent sexual development is a welcome addition to contemporary theorizing about adolescent sexuality. It draws productively from existing strains of research on positive adolescent sexual development [Bay-Cheng, 2012; Fine \& McClelland, 2006; Impett, Muise, \& Breines, 2013; Lamb, 2010; Russell, 2005; Tolman, 2012; Tolman \& McClelland, 2011] but breaks new ground in its more specific and process-oriented approach. Whereas previous scholars have effectively outlined the need for and the promise of positive developmental models of adolescent sexuality, Arbeit has taken on the thorny details, outlining a vision of the fundamental components of positive adolescent sexuality (sexual selfhood, sexual negotiation, and sexual empowerment) and the psychosocial skills that they require. A particular strength of the model is the proactive role that it grants to adolescents themselves as drivers of their own development. In contrast to the hundreds of studies that focus on how adolescent sexuality is shaped by outside forces (such as parents, peers, teachers, school, their church, the media, the Internet, and the culture at large), Arbeit's model casts adolescents themselves as the central characters in their own emerging sexual selfhood. In her conceptualization, youths are not simply passive actors, consuming conflicting messages from parents, peers and the media, and moving back and forth with changing cultural tides, but active shapers of their own sexual-developmental trajectories. This allows researchers to ask far more sophisticated questions about the dynamic interchanges between youths and their environments that produce variability in sexualdevelopmental pathways and outcomes.

I am enthusiastic about Arbeit's model and its potential to generate important new questions and answers about adolescent sexual development. Nonetheless, there are "soft spots" in the model that could benefit from greater attention, clarification, and expansion. My discussion of these areas below is intended to enhance and improve the impact and generativity of Arbeit's model rather than to challenge it. She has charted a new course in the study of adolescent sexuality, and working to test and refine this new course should be the work of all scholars of adolescent sexuality.

\section{Expanding the Frame: The Need for a Cumulative Life Span Approach}

Arbeit grounds her model in relational developmental systems theory [Lerner, Lerner, Almerigi, \& Theokas, 2006; Overton, 2013], which views human development as the evolving product of an ongoing series of dynamic person-context relations. Yet this perspective raises an inevitable question: why restrict a model of sexual development to adolescence? Although the developmental transitions of adolescence arguably make it a "critical period" for sexual development, sexuality is obviously a lifelong experience. Furthermore, a growing body of theoretical models related to developmental systems theory have called for "cascade" models of human development [Lansford, Malone, Dodge, Pettit, \& Bates, 2010; Masten \& Cicchetti, 2010; Ryff, Singer, \& Seltzer, 2002; Ryff, Singer, Wing, \& Love, 2001], in which each developmental phenomenon is conceptualized as both the output of prior developmental processes and the input for forthcoming processes. Hence, from this perspective it may be im- 
possible to study positive adolescent sexual development without beginning with a focus on childhood sexual experiences, cognitions, and skills. Arbeit is clearly aware of the relevance of childhood processes and indicates that sexual skill development begins with children's own bodily exploration and early childhood interactions, but these childhood experiences receive little explicit attention in her model, nor do the potential sexual-developmental implications of early childhood adversity, neglect, or abuse. More comprehensive integration of childhood sexual development into Arbeit's model would substantially expand its reach.

Similarly, it would appear to be impossible to differentiate positive versus negative trajectories of sexual development during adolescence without extending the time frame forward substantially into middle and late adulthood. After all, research has shown that many adult sexual problems and dysfunctions are related to interpersonal and situational stressors occurring at all stages of the life course [Laumann, Paik, \& Rosen, 1999]. Hence, there is no particular age at which we can confidently establish that a youth has succeeded in developing a healthy, positive, sexual self. Rather, positive sexual development is a life span task which requires a life span research frame and an investigative approach capable of attending to the cumulative, cascading processes that give rise to dynamic changes in sexual selfhood across different ages and contexts.

Arbeit's model is well-suited to take this approach: she indicates that the hypothesized developmental processes outlined in figure 1 are likely to recur many times over, resulting in the development and refinement of different sets of skills across an expanding range of contexts. Surely, these recurrences may occur over the entire life span, as developmental and contextual changes create new needs and challenges. Consider, for example, the skills required for effective sexual negotiation. As Arbeit indicates, the development of skills related to verbalizing one's needs and boundaries, establishing and negotiating consent, and successfully seeking and providing pleasure are critically important during the adolescent years, but they may require substantial refinement and elaboration at many different stages of the life span as individuals encounter new sexual partners with different expectations, different bodies, different patterns of communication, and different sexual tastes. Aging, too, creates new challenges: consider two individuals in their 70s dating for the first time, struggling to find an artful way to describe and work around potential physical and/or health limitations to their sexual behavior. No matter how successfully they might have mastered sexual negotiation in previous decades, the new developmental challenges accompanying later stages of life create new challenges and the need for new sets of skills. Similarly, imagine a married couple coping with the reestablishment of sexual intimacy after one partner's infidelity. The challenges of this novel context might tax their skills of sexual negotiation in new and unexpected ways.

Hence, as long as individuals undergo lifelong psychosocial, biological, emotional, cognitive, and ideological changes, and as long as they move into and out of different relationships and contexts, they will inevitably repeat and revisit Arbeit's hypothesized processes of sexual development. Accordingly, one of the most generative implications of Arbeit's perspective is that it could be used as the foundation for a truly life span model of sexual development. Although adolescence might prove to be a particularly critical period for the development of sexual skills and sexual selfhood, it is clearly not the only one. Rather, adolescent sexuality represents one stage in a cascade model - sexual feelings, experiences, skills, and identities during the 
adolescent years are both the outcome of childhood feelings, experiences, skills, and identities, and also the drivers of feelings, experiences, skills, and identities during early, middle, and late adulthood. Although Arbeit does not substantively integrate this cascade perspective into her model, it is fully consistent with her emphasis on relational developmental systems theory.

\section{Expanding the Focus on Biological Processes}

Another area of Arbeit's model which would profit from expansion and elaboration concerns the role of biological processes. Although Arbeit makes several references to the influence of biological factors on adolescent sexual development, these references are relatively vague, and the overall role of biological processes in her model is underdeveloped. If, as she states, her goal is to draw greater attention to the systematic relations involved in promoting positive sexual development, then biology deserves a larger and more specific role. Furthermore, greater focus on the biological body would enhance Arbeit's commendable emphasis on embodiment as a critical component of sexual development. As Arbeit reviews, the concept of embodiment encapsulates the fact that youths' sexual development, their sense of sexual selfhood, and their development as sexual citizens is negotiated by, with, and through their bodies following the influential work of Overton [2008] and Tolman, Bowman, and Fahs [2014]. Although research on embodiment typically focuses on the subjective phenomenology of bodily experience, it can and should include a focus on the many systems within the body which undergo change before, during, and after sexual maturation. Arbeit highlights that the development of sexuality-related skills occurs within young people's bodies and in relation to "past and future bodies." During a time of such incredible physical change, these past and future bodies might be astoundingly different, both in obvious and not-so-obvious ways. Although an adolescent (and his or her peers, parents, and potential sexual partners) might be acutely aware of bodily changes signaling sexual maturation (height, hair growth, breast size, hip size, muscular development), they are probably less aware of invisible changes such as those related to brain structure and function and to autonomic and neuroendocrine functioning. Yet these changes undoubtedly interact in critical ways with adolescents' other social and sexual transitions, and their implications for youths' sense of embodied sexuality deserve greater attention. Hence, one provocative and potentially transformative direction for Arbeit's model would incorporate a more thoroughgoing conceptualization of "the body," in all of its interrelated systems and processes, to provide a more biologically specific understanding of sexual development.

Consider, for example, the role of hormonal factors. Arbeit makes general reference to the role of hormones in the development of sexual desire, but the complex relationship between hormones and sexual desire merits more detailed and specific attention within a contextual model such as Arbeit's. For example, in addition to the well-known changes in gonadal hormones that youths undergo during pubertal maturation, there are also substantive increases in adrenal androgens such as dehydroepiandrosterone, dehydroepiandrosterone sulfate, and androestione, which occur during the maturation of the adrenal gland between 6 and 10 years of age, and some scholars have argued that these hormonal changes may be responsible for the fact that many adults recollect their first erotic crushes and thoughts as occurring around the age of 
10 [Herdt \& McClintock, 2000]. Hence, understanding the different psychological sequelae of adrenal versus gonadal androgens is important for understanding youths' emerging and changing experiences of sexual arousal from childhood to adolescence.

Similarly, van Anders [2012] has found that the hormonal underpinnings of dyadic desire (i.e., desire for sexual activity and release with another person) differ significantly from the hormonal underpinnings of solitary desire (i.e., desire for sexual release without a partner). Although most research fails to even acknowledge this distinction, van Anders' work has shown that, in adults, the distinction has important biobehavioral implications. Specifically, she found that women's testosterone levels were positively linked to their solitary desires and that masturbation frequency moderated this link. Yet testosterone levels were negatively correlated with women's $d y$ adic desires. These provocative findings suggest that the developmental transition from desiring solitary sexual release to desiring partnered sexual release may have hormonal as well as social and cultural determinants and warrants longitudinal investigation before, during, and after pubertal maturation. For example, one possibility is that the very distinction between solitary and dyadic desire may only become meaningful after puberty due to the changing levels of gonadal hormones. Another possibility is that the subjective experiences of these two types of desire change substantially during the pubertal transition. These examples demonstrate the potential value of a more specific integration of biological processes into Arbeit's model.

Neurobiological development is another growing area of research that deserves more substantive attention. There is now an extensive body of neurobiological research on sexuality (most commonly using functional magnetic resonance imaging, but also positron emission tomography, and, less often, electroencephalography), and much of this research bears on the question of overlap between the neurobiological bases for romantic love, emotional attachment, and sexual arousal [reviewed in Diamond \& Dickenson, 2012]. Studies of adults suggest that romantic love and sexual arousal are neither neurobiologically independent nor redundant but show complex patterns of linkage that may be related to their experiential contexts. This raises provocative questions regarding developmental changes before and during the adolescent years, when youths' capacities for sexual desire and romantic attachment undergo notable changes. Might developmental changes in the brain structures involved in sexual desire and romantic attachment - and their interconnectedness - relate to developmental changes in youths' subjective experiences of sex and love and their motives for emotional and physical intimacy? Investigating such questions from a developmentalsystems perspective (in which biological changes interact fundamentally with contextual factors) can make tremendous contributions to the basic developmental science of sexuality and can elucidate the degree to which other aspects of neural development (for example, maturation in structures related to decision-making, future-oriented thinking, self-regulation) interact with the brain regions involved in sexuality to shape youths' trajectories of sexual behavior and other behaviors.

Finally, Arbeit's model would profit from greater integration with the "differential susceptibility" model of Belsky and Pluess [2009] and the related "biological sensitivity to context" model of Boyce and Ellis [2005]. According to these models, children and adolescents with heightened autonomic and neuroendocrine reactivity to stress are differentially sensitive to both the negative and positive features of their social-environmental contexts. This model has its roots in the well-known diathesisstress model of development, which predicts that underlying vulnerability factors 
confer heightened risk for poor adjustment outcomes when combined with environmental challenges. Yet differential susceptibility models additionally predict that the same factors that predispose certain youths to heightened risk within suboptimal contexts also predispose youths to heightened benefits within enriched and nurturant contexts. Such models may prove particularly powerful for elucidating the factors driving diversity in youths' sexual-developmental trajectories. For example, differential susceptibility models would predict that autonomically reactive adolescents are disproportionately likely to develop adjustment problems after experiencing a sequence of negative and disempowering sexual experiences but that the very same youths are disproportionately likely to develop heightened self-esteem and an enhanced sense of sexual agency after experiencing a sequence of positive, pleasurable, and empowering sexual experiences.

Most of the extant research testing differential susceptibility models focuses on young children, yet testing such models among adolescents is important because the neurobiological systems underlying stress regulation are still undergoing maturation during this period [Dahl \& Spear, 2004], and the emotional and self-regulatory challenges and opportunities posed by adolescent sexual desire and behavior likely place heightened demands on these systems. Hence, greater assessment of individual differences in youths' physiological stress reactivity, in concert with detailed assessment of their early rearing environments and their current environmental contexts, has the potential to reveal long-range patterns of adaptation and adversity that help to explain how different youths may undergo similar sexual experiences in adolescence and yet show markedly different adjustment outcomes. In particular, research investigating positive and negative family and peer influences on adolescent sexuality would profit by using differential susceptibility models to investigate which youths are most susceptible to such influences and how this information can be integrated into attempts to develop health-promoting interventions.

\section{Grappling with Gender Variation}

A strength of Arbeit's model is her multifaceted conceptualization of sexual selfhood, which involves awareness of one's desires, adherence to one's sense of sexual ethics, and the process of "grappling" with sexual identity development. I would argue that sexual selfhood also necessarily involves grappling with one's gender identity and that this aspect of sexual development requires further elaboration within Arbeit's model. Just as healthy sexual selfhood requires that youths wrestle with and interrogate their beliefs, cognitions, and phenomenology regarding sexual desire, it also requires wrestling with and interrogating their beliefs, cognitions, and phenomenology regarding femaleness and maleness.

A growing body of research challenges the notion that gender identity finishes developing in early childhood and indicates that many youths actively question their subjective experience and presentation of gender during childhood and adolescent years [reviewed in Diamond, 2013a]. One large-scale study of 6- to 10-year-old children [Sandberg, Meyer Bahlburg, Ehrhardt, \& Yager, 1993] found that approximately $23 \%$ of boys and $39 \%$ of girls showed 10 or more different gender-nonconforming behaviors. It is difficult to make inferences about the prevalence of adolescent gender nonconformity from these data, however, because there is evidence that, as children 
grow older and begin to perceive the social implications of gender nonconformity, they consciously modify their own behavior, sometimes becoming extremely gender stereotyped in order to avoid and compensate for prior stigmatization [Hunter, 1993]. Other gender-nonconforming adolescents may adopt transgender identities, which may entail adopting a cross-gender identification, an intermediate or dualgender presentation, or the rejection of gender as a relevant identity category altogether [Denny, 2004; Diamond, Pardo, \& Butterworth, 2011; Mallon \& DeCrescenzo, 2006].

Because mainstream culture continues to presume (and to communicate through various media sources) that gender nonconformity is a sign of homosexuality, adolescents may find it impossible to question their gender identity and performance without also questioning their sexual orientation and identity and vice versa. Hence, Arbeit's focus on the dynamic psychological and interpersonal processes driving sexual development would be greatly enhanced by greater attention to the interplay between gender and sexual questioning and how it plays out differently for youths with different constellations of "atypical" desires and atypical gender presentation. One reason to adopt this expanded focus is to enhance our basic understanding of the psychosexual development of transgender and gender-nonconforming (sometimes called gender-variant) adolescents more broadly. In contrast to the extensive body of research on conventional gender identity development [Ruble, Taylor, Cyphers, Greulich, Lurye, \& Shrout, 2007], little research has focused on the basic developmental processes of transgender identity development in nonclinical populations [Gagné, Tewksbury, \& McGaughey, 1997]. Historically, psychologists have expressed ambivalence about whether it is healthy for youths to embrace permanently liminal, flexible senses of gender instead of moving progressively toward the goal of consistently identifying as male or female [reviewed in Mallon \& DeCrescenzo, 2006], yet Arbeit's model provides a framework for reconceptualizing gender questioning as adaptive and even normative.

Specifically, to the extent that Arbeit's model suggests that healthy sexual development requires that youths actively interrogate, interpret, take responsibility for, and take ownership of their personal experiences of desire, perhaps healthy gender development similarly requires that youths actively interrogate, interpret, take responsibility for, and take ownership of their personal experience of gender. Similarly, to the extent that these agentic processes contribute to the development of a sexual ethics, they might similarly contribute to the development of "gender ethics" in which youths come to develop values and personal standards regarding all human beings' entitlement to dignity, integrity, and autonomy regarding their subjective experience of gender. Perhaps by fostering such an appreciation for "gender dignity" and "gender ethics," we may be able to reduce youths' tendency to victimize and marginalize peers who fall short of the culture's restrictive standards regarding "correct" and/or "normal" female and male behavior.

\section{Gender as a Product of Sexuality}

Another aspect of Arbeit's model which would benefit by more substantive attention to gender concerns the process of sexual negotiation. She aptly identifies sexual negotiation as a foundational sexual skill, but may not fully appreciate the 
role that these negotiations play in shaping youths' developing sense of gender as an interactional phenomenon. Because our culture rewards young men and women differently for expressing their feelings, asserting their goals, and exerting versus resisting influence, girls and boys will inevitably enter into sexual negotiations with gender-differentiated patterns of cognition and behavior. Yet a relational-developmental-systems perspective calls our attention to the fact that the dynamic links between gender and sexual negotiation are necessarily bidirectional. In other words, just as girls with certain expectations about "appropriate" female behavior will enter into sexual negotiations with certain biases and constraints, girls will also exit each such negotiation with updated beliefs, expectations, and feelings regarding their gender. In essence, gender does not simply influence adolescent sexual experience, it is also dynamically produced by adolescent sexual experience. For example, a girl who experiences a series of successes in negotiating tricky sexual interactions is likely to develop a sense of womanhood that is fully consistent with autonomy, need assertion, and feelings of competency and dignity in claiming one's right to pleasure and to one's own bodily boundaries. A girl whose sexual negotiations end poorly, and who finds herself the subject of blame, pressure, and shame, may develop a sense of womanhood that requires subjugating one's own will to male privilege and authority. Investigating such processes would expand the reach and significance of Arbeit's model and would permit a broader understanding of how youths begin to experience larger structures of power and privilege through the proximal context of dyadic sexual interactions.

\section{Greater Attention to Sexual-Minority Development}

A strength of Arbeit's model is that she does not posit fundamentally different sexual-developmental processes for heterosexual versus sexual-minority (i.e., samesex attracted) youth. Yet this inclusive approach risks losing sight of unique issues related to sexual orientation and identity that would enhance the applicability and scope of her model. For example, to the extent that the negative slant of previous research on adolescent sexuality has hindered our attempts to develop positive, healthenhancing interventions for youths, this weakness has been compounded for sexualminority youths whose sexual feelings and needs have been viewed as even more dangerous, threatening, and potentially harmful than those of heterosexuals. For example, although there has been an impressive proliferation of outreach efforts (at the level of advocacy organizations, clinicians, teachers, etc.) aimed at addressing the psychosocial needs of sexual-minority youths, these efforts often take great care to avoid any impression that they are encouraging youth with same-sex attractions to act on their attractions and, instead, generally focus on "safe" issues such as the prevention of suicidality and antigay bullying. Hence, although society has reached consensus that sexual-minority youth should not face psychological or physical harm, it has not reached consensus on whether it is okay for these youths to actually express their sexuality.

Arbeit's model has the potential to provide a powerful counterargument to this myopic approach. Her skill-based model suggests that we cannot expect youths to develop into ethical, responsible, positive, healthy sexual citizens if we deny them the opportunities to practice the critical skills of assessing, experiencing, analyzing, shar- 
ing, negotiating, and enjoying their sexual feelings, cognitions, and sensations. One might interpret Arbeit's model to suggest that it is impossible to foster healthy psychosocial development among sexual-minority youths if the very basis for their social marginalization - same-sex desire - is denied expression. Hence, antibullying efforts and gay-straight alliances must be undertaken in concert with efforts to provide sexual-minority youths with safe spaces to discuss, think about, and act on their samesex desires, with ready access to the guidance, advice, and support of trusted adults (given that many such youths are alienated from their parents). Developing a sense of autonomy and sexual agency is critical for all youths but may prove even more important for youths whose sexuality is marginalized and denigrated by the culture at large.

Another reason that Arbeit's model would profit by greater attention to questions of same-sex sexuality, and the unique stigmatization that it engenders in contemporary culture, is the simple fact that there is such greater variability in the expression of same-sex and other-sex desire and behavior during the adolescent years. The National Longitudinal Study of Adolescent Health found that 13\% of American girls and $6 \%$ of American boys reported same-sex attractions, same-sex relationships, or nonheterosexual identities [Savin-Williams, 2005]. The National Survey of Family Growth, with a representative national sample of nearly 14,000 American individuals, found that $5.2 \%$ of male teenagers and $12.5 \%$ of female teenagers reported same-sex sexual contact [Chandra, Mosher, Copen, \& Sionean, 2011]. Importantly, longitudinal research indicates that same-sex behavior in adolescence is not necessarily a precursor to a sexual-minority identity. Savin-Williams and Ream [2007] found that, among the adolescents in the National Longitudinal Study of Adolescent Health who reported same-sex behavior at wave 1 (at a mean age of 16), very few ( 1 in 5 females and 1 in 25 males) described themselves as "homosexual," "mostly homosexual," or "bisexual" by wave 3 (at a mean age of 22), and the overall percentage of youths adopting one of these descriptors was low (2.4\% of males and $3.8 \%$ of females). Similarly, in the National Survey of Family Growth, $2.8 \%$ of 18 - to 19 -year-old males and $7.7 \%$ of 18- to 19-year-old females identified themselves as gay, lesbian, or bisexual [Chandra et al., 2011]. These findings accord with other research conducted with large samples of adolescents, indicating that the vast majority of youth reporting same-sex attractions or same-sex behavior present themselves as heterosexual [Garofalo, Wolf, Wissow, Woods, \& Goodman, 1999; Mosher, Chandra, \& Jones, 2005; Remafedi, Resnick, Blum, \& Harris, 1992].

Hence, although many researchers conceptualize adolescents as falling into two rough groups - a heterosexual group that shows no interest in same-sex activity and a gay and lesbian group that shows no interest in other-sex activity - this is simply inaccurate. Accordingly, we need models of adolescent sexual development capable of representing the incredible diversity of youths' trajectories of same-sex and othersex desire and behavior during the adolescent years and which do not presume that the frequency, timing, or quality of these experiences necessarily predicts later sexual identification. The emphasis that Arbeit places on youths' autonomy, agency, and active construction of sexual selfhood renders her model well suited to make important contributions to our current understanding of the development of sexual orientation and identity during the adolescent years, and this remains one of the most promising applications of her model. 


\section{The Meaning of Meaning-Making}

A final area deserving of greater elaboration in Arbeit's model, and greater integration of a cascade approach, is the notion of sexual meaning-making and the process through which youths continually craft and revise their own sexual autobiographies. In Arbeit's model, meaning-making only receives specific attention in the context of sexual ethics, as youths reflect on and interpret their own personal experiences and come to apply the resulting insights to novel events [Smetana, 2006]. The critical role of meaning-making in sexual ethics derives from the fact that sexual ethics requires that youths sift through and evaluate their own experiences against the multiple, potentially competing messages communicated by parents, peers, the media, teachers, and church leaders in order to arrive at their own sense of sexual values and decision-making. Although Arbeit is right to place emphasis on the process of meaning-making in the context of the development of sexual ethics, she perhaps underestimates the critical role of meaning-making in sexual development more broadly and does not devote sufficient attention to its critical role within a dynamic, cascade approach to sexual development.

For example, given her nuanced conceptualization of sexual desire as an experience which requires self-reflection and assessment of one's own physical, emotional, and cognitive reactions, meaning-making would appear to be central to this process. In other words, the very process of embodiment requires an interpretive framework. For youths to assess their subjective, embodied experiences and apply these assessments to their ongoing skill development, chronic processes of meaning-making must inevitably occur. In this respect, the growing body of research on autobiographical memory and narrative could potentially play an important role in expanding Arbeit's focus and providing new avenues for investigation. Specifically, when we think of the development of sexual selfhood, we would profit by conceiving of the sexual self as a narrative construction, one that is actively composed and revised over time in the context of changing motives and experiences and in light of youths' expanding base of knowledge and personal experience.

A greater focus on autobiographical narrative and meaning-making is particularly relevant to cascade models of sexual development in which a youth's present subjective experience within a sexual interaction is fundamentally structured by the meanings attached to similar, previous experiences and feeds forward to shape future expectations, interpretations, and meanings. This process has great significance for adaptation and psychological growth: previous research has found that crafting meaningful understandings of both positive and negative life experiences helps individuals to cope with and learn from stressors and challenges, particularly if individuals use their experiences to derive insights that can guide their future choices and self-knowledge [McLean \& Breen, 2009; McLean, Breen, \& Fournier, 2010; McLean \& Pasupathi, 2007]. Hence, youths who craft meaningful insights from stressful or disappointing sexual experiences, and who make an effort to determine what to do in the future to reduce the likelihood of such experiences, may actually show enhanced development relative to youths who cope with negative experiences by dismissing the thoughts and feelings they provoke.

Greater attention to the cascading role of meaning-making may help to make sense of the mixed patterns of association that have been detected between sexual experience and adolescent well-being, with some studies showing greater happiness and 
self-esteem among more sexually experienced girls [Horne \& Zimmer-Gembeck, 2005] and others showing the opposite [Ciairano, Bonino, Kliewer, Miceli, \& Jackson, 2006]. The subjective quality and interpretation of youths' experiences may prove to be the key unmeasured variable. Hence, one potential extension of Arbeit's model might involve greater emphasis on how youths' motives, expectations, and interpretations of their successive sexual and romantic experiences shape their unfolding sexual-developmental trajectories over the course of adolescence in a cascading fashion such that youths' meaning-making processes drive future sexual decisions and experiences, and these experiences then feed forward to shape future meaning-making processes. This approach may allow us to investigate how and why youths transition from one type of sexual experience to another over the course of adolescence and how these experiences shape the overall development of their particular constellation of sexual desires, preferences, needs, fears, etc. Information on cascading relations between youths' experiences and their interpretations of these experiences is also particularly useful for understanding cases in which youths' trajectories change course, shifting from positive to negative experiences and vice versa, and such information is likely to be critically important for the development of health-promoting interventions for adolescents. Specifically, understanding and altering the types of interpretive frameworks that youths apply to both positive and negative sexual experiences may help to foster their sense of agency, help them to take ownership of their sexual phenomenology, and empower them to make positive changes in their emerging sexual selves.

\section{Conclusion}

It bears noting that my critiques and suggested extensions of Arbeit's model testify to its utility. Importantly, Arbeit states clearly that her goal is not to provide a uniform "answer" to promoting positive sexual development in adolescence but to provide a generative roadmap to the types of questions and research agendas that might prove most fruitful. In this she has undoubtedly succeeded. Even her open acknowledgement of pleasure as a fundamental component of adolescent sexual development is strikingly novel. A recent search of the published psychological literature found over 3,000 journal articles with the words "adolescent/ce" and "sexual/sexuality" in the title, but only 31 included the word "pleasure" in the title or abstract. This reflects our culture's chronic ambivalence about sexual pleasure and its historical connotation with immorality and self-indulgence. Yet in Arbeit's model, pleasure plays an ever-present and fundamental role not only as a contributor to sexual needs and behaviors but also as a developmental achievement in and of itself with respect to youths' capacities to identify and enjoy sensations and feelings and link them up with a sense of fundamental entitlement to pleasure. Accordingly, her model has great potential to provide a much-needed corrective to the chronic sex negativity which has hindered models of adolescent sexuality as well as models of adult health and relationships [Diamond, 2013b; Diamond \& Huebner, 2012]. The areas for potential expansion that I have highlighted simply indicate the broad applicability of her dynamic, skill-based approach and its potential to generate important new lines of inquiry into life span sexual development. 


\section{References}

Bay-Cheng, L.Y. (2012). Recovering empowerment: De-personalizing and re-politicizing adolescent female sexuality. Sex Roles, 66, 713-717.

Belsky, J., \& Pluess, M. (2009). Beyond diathesis stress: Differential susceptibility to environmental influences. Psychological Bulletin, 135, 885-908.

- Boyce, W.T., \& Ellis, B.J. (2005). Biological sensitivity to context. I. An evolutionary-developmental theory of the origins and functions of stress reactivity. Development and Psychopathology, 17, 271-301.

Chandra, A., Mosher, W.D., Copen, C., \& Sionean, C. (2011). Sexual behavior, sexual attraction, and sexual identity in the United States: Data from the 2006-2008 National Survey of Family Growth. National Health Statistics Reports, March 3, 1-36.

-Ciairano, S., Bonino, S., Kliewer, W., Miceli, R., \& Jackson, S. (2006). Dating, sexual activity, and wellbeing in Italian adolescents. Journal of Clinical Child and Adolescent Psychology, 35, 275-282.

Dahl, R.E., \& Spear, L.P. (2004). Adolescent brain development: Vulnerabilities and opportunities. New York, NY: New York Academy of Sciences.

Denny, D. (2004). Changing models of transsexualism. In U. Leli \& J. Drescher (Eds.), Transgender subjectivities: A clinician's guide (pp. 25-40). New York, NY: Haworth Press.

Diamond, L.M. (2013a). Sexual-minority, gender-nonconforming, and transgender youths. In D.S. Bromberg \& W.T. Donohue (Eds.), Handbook of child and adolescent sexuality: Developmental and forensic psychology (pp. 275-300). Oxford, England: Elsevier Press.

Diamond, L.M. (2013b). Where's the sex in relationship research? In R.S. Stewart (Ed.), Let's talk about sex: Multidisciplinary discussions (pp. 48-68). Sydney, Nova Scotia: Cape Breton University Press.

Diamond, L.M., \& Dickenson, J.A. (2012). The neuroimaging of love and desire: Review and future directions. Clinical Neuropsychiatry, 9, 39-46.

Diamond, L.M., \& Huebner, D.M. (2012). Is good sex good for you? Reconsidering sexuality and health. Social and Personality Psychology Compass, 6, 54-69.

Diamond, L.M., Pardo, S.T., \& Butterworth, M.R. (2011). Border crossings: Transgender experience and identity. In S. Schwartz, K. Luyckx, \& V. Vignoles (Eds.), Handbook of identity theory and research (pp. 629-648). New York, NY: Springer.

Fine, M., \& McClelland, S.I. (2006). Sexuality education and desire: Still missing after all these years. Harvard Educational Review, 76, 297-338.

Gagné, P., Tewksbury, R., \& McGaughey, D. (1997). Coming out and crossing over: Identity formation and proclamation in a transgender community. Gender \& Society, 11, 478-508.

Garofalo, R., Wolf, R.C., Wissow, L.S., Woods, E.R., \& Goodman, E. (1999). Sexual orientation and risk of suicide attempts among a representative sample of youth. Archives of Pediatrics and Adolescent Medicine, 153, 487-493.

Herdt, G., \& McClintock, M. (2000). The magical age of 10. Archives of Sexual Behavior, 29, 587-606.

Horne, S., \& Zimmer-Gembeck, M.J. (2005). Female sexual subjectivity and well-being: Comparing late adolescents with different sexual experiences. Sexuality Research \& Social Policy: A Journal of the NSRC, 2, 25-40.

Hunter, A. (1993). Same door, different closet: A heterosexual sissy's coming-out party. In S. Wilkinson \& C. Kitzinger (Eds.), Heterosexuality: A feminism and psychology reader (pp. 150-168). London, England: Sage.

Impett, E.A., Muise, A., \& Breines, J.G. (2013). From risk to pleasure: Toward a positive psychology of sexuality. In M. Hojjat \& D. Cramer (Eds.), Positive psychology of love (pp. 57-75). New York, NY: Oxford University Press.

Lamb, S. (2010). Feminist ideals for a healthy female adolescent sexuality: A critique. Sex Roles, 62, 294306.

Lansford, J.E., Malone, P.S., Dodge, K.A., Pettit, G.S., \& Bates, J.E. (2010). Developmental cascades of peer rejection, social information processing biases, and aggression during middle childhood. Development and Psychopathology, 22, 593-602.

Laumann, E.O., Paik, A., \& Rosen, R.C. (1999). Sexual dysfunction in the United States: Prevalence and predictors. Journal of the American Medical Association, 281, 537-544.

Lerner, R.M., Lerner, J.V., Almerigi, J., \& Theokas, C. (2006). Dynamics of individual-context relations in human development: A developmental systems perspective. In J.C. Thomas, D.L. Segal, \& M. Hersen (Eds.), Comprehensive handbook of personality and psychopathology. Vol. 1: Personality and everyday functioning (pp. 23-43). New York, NY: John Wiley \& Sons.

Mallon, G.P., \& DeCrescenzo, T. (2006). Transgender children and youth: A child welfare practice perspective. Child Welfare Journal, 85, 215-241.

-Masten, A.S., \& Cicchetti, D. (2010). Developmental cascades. Development and Psychopathology, 22, 491-495. 
McLean, K.C., \& Breen, A.V. (2009). Processes and content of narrative identity development in adolescence: Gender and well-being. Developmental Psychology, 45, 702-710.

McLean, K.C., Breen, A.V., \& Fournier, M.A. (2010). Constructing the self in early, middle, and late adolescent boys: Narrative identity, individuation, and well-being. Journal of Research on Adolescence, 20, 166-187.

McLean, K.C., \& Pasupathi, M. (2007). Selves creating stories creating selves: A process model of selfdevelopment. Personality and Social Psychology Review, 11, 262-278.

Mosher, W.D., Chandra, A., \& Jones, J. (2005). Sexual behavior and selected health measures: Men and women 15-44 years of age, United States, 2002 (pp. 1-56). Advance data from vital and health statistics, No. 362. Hyattsville, MD: National Center for Health Statistics.

Overton, W.F. (2008). Embodiment from a relational perspective. In W.F. Overton, U. Müller, \& J.L. Newman (Eds.), Developmental perspectives on embodiment and consciousness (pp. 1-18). New York, NY: Taylor \& Francis/Lawrence Erlbaum.

Overton, W.F. (2013). A new paradigm for developmental science: Relationism and relational-developmental systems. Applied Developmental Science, 17, 94-107.

Remafedi, G., Resnick, M., Blum, R., \& Harris, L. (1992). Demography of sexual orientation in adolescents. Pediatrics, 89, 714-721.

Ruble, D.N., Taylor, L.J., Cyphers, L., Greulich, F.K., Lurye, L.E., \& Shrout, P.E. (2007). The role of gender constancy in early gender development. Child Development, 78, 1121-1136.

Russell, S.T. (2005). Conceptualizing positive adolescent sexuality development. Sexuality Research \& Social Policy: A Journal of the NSRC, 2, 4-12.

Ryff, C.D., Singer, B.H., \& Seltzer, M.M. (2002). Pathways through challenge: Implications for well-being and health. In L. Pulkkinen \& A. Caspi (Eds.), Paths to successful development: Personality in the life course (pp. 302-328). New York, NY: Cambridge University Press.

Ryff, C.D., Singer, B.H., Wing, E., \& Love, G.D. (2001). Elective affinities and uninvited agonies: Mapping emotion with significant others onto health. In C.D. Ryff \& B.H. Singer (Eds.), Emotions, social relationships, and health (pp. 133-174). New York, NY: Oxford University Press.

Sandberg, D.E., Meyer Bahlburg, H.F., Ehrhardt, A.A., \& Yager, T.J. (1993). The prevalence of genderatypical behavior in elementary school children. Journal of the American Academy of Child and Adolescent Psychiatry, 32, 306-314.

Savin-Williams, R.C. (2005). The new gay teenager. Cambridge, MA: Harvard University Press.

Savin-Williams, R.C., \& Ream, G.L. (2007). Prevalence and stability of sexual orientation components during adolescence and young adulthood. Archives of Sexual Behavior, 36, 385-394.

Smetana, J.G. (2006). Social-cognitive domain theory: Consistencies and variations in children's moral and social judgments. In M. Killen \& J.G. Smetana (Eds.), Handbook of moral development (pp. 119-153). New York, NY: Taylor \& Francis.

Tolman, D.L. (2012). Female adolescents, sexual empowerment and desire: A missing discourse of gender inequity. Sex Roles, 66, 746-757.

Tolman, D.L., Bowman, C.P., \& Fahs, B. (2014). Sexuality and embodiment. In D.L. Tolman, L.M. Diamond, J.A. Bauermeister, W.H. George, J.G. Pfaus, \& L.M. Ward (Eds.), APA handbook of sexuality and psychology. Vol. 1: Person-based approaches (pp. 759-804). Washington, DC: American Psychological Association.

Tolman, D.L., \& McClelland, S.I. (2011). Normative sexuality development in adolescence: A decade in review, 2000-2009. Journal of Research on Adolescence, 21, 242-255.

Van Anders, S.M. (2012). Testosterone and sexual desire in healthy women and men. Archives of Sexual Behavior, 41, 1471-1484.

World Health Organization (2004). Sexual health: A new focus for WHO. Progress in Reproductive Health Research, 67, 1-8. 